\title{
OpenESSENCE: Disease Surveillance through Medical Record System Integration with OpenMRS
}

\author{
Charles Hodanics*
}

JHU APL, Laurel, MD, USA

\section{Objective}

By pairing an open source medical record system, OpenMRS, with the Suite for Automated Global Electronic bioSurveillance (SAGES) disease surveillance tool, OpenESSENCE (OE), we have prototyped an open source solution for passive disease and program surveillance using an active medical record system.

\section{Introduction}

OpenMRS has global presence as an open source web based medical record system (MRS). It is built on an extensible, modular framework that allows the user to create a MRS that is as simple or complex as needed.

OE is a multi-user network accessible analysis and visualization tool that enables users to monitor the population's health from any computer connected to that network. OE was created as an open source solution using features and lessons learned from Enterprise ESSENCE, (Electronic Surveillance System for Early Notification of Community-Based Epidemics.) OE provides analyses, maps, graphs, charts, crosstabs, as well as detail tables and export functionality.

\section{Methods}

Open source tools now cover a wide spectrum of domains with numerous solutions for any given problem. Deciding how to integrate a MRS with OE involved evaluating three integration paths: 1) building a MRS into OE; 2) building disease surveillance into a MRS; and 3) pairing an existing MRS with OE. Several open source MRS tools exist and after reviewing the tools for functionality and compatibility with SAGES and specific project criteria, pairing OpenMRS with OE was the optimal approach. OpenMRS, when compared with other open source MRS tools, had the ideal license, platform, target use case and community support, and was therefore selected as the open source tool for integration.

The approach taken to pair OpenMRS patient level data with OE stemmed from a core design principle in $\mathrm{OE}$ - providing data agnostic analysis through compatibility with existing data structures. OpenMRS has a complex data structure that allows for data extensibility from the website front end, and as such, stores data in a highly normalized way. OE is most easily configured to run analysis on a de-normalized data structure. In addition to flattening the complex data structure that OpenMRS uses, the internationalization inherently supported by the OpenMRS data structure was accounted for in OE. The modifications provide the capability of supporting OpenMRS coded data values that have translations in the OE query and analysis capabilities.

\section{Results}

OE has successfully been configured to read OpenMRS data. This pairing allows OE to provide analysis and visualization on data collected through OpenMRS clinic visits. Using the OpenMRS data framework by sharing the underlying data structure allows end users of the system to easily add new data elements to OpenMRS, include the new element into the OpenMRS patient data collection system, and configure $\mathrm{OE}$ to recognize the new element for reports and analysis. This cycle can be performed through the OpenMRS and OE websites as needed to modify or augment data collection and disease surveillance capabilities, allowing the system to adapt as the environment grows.

\section{Conclusions}

Integration of the OE and OpenMRS software applications is a novel step towards providing the enhanced capabilities of larger disease surveillance systems to resource limited environments. This pairing combines two extensible systems with open source support that fill complementary needs in an overlapping domain, providing a flexible solution that can begin very simply and grow as user needs and experiences grow.

By leveraging the strengths of both tools - one focused on patient and programmatic data and another focused on analytics and disease surveillance - a more complete system can be realized.

\section{Keywords}

Opensource; Medical Record System; Disease Surveillance; OpenESSENCE

\section{Acknowledgments}

Charles Hodanics, Jacqueline Coberly, Vivian Hung, Colin Taylor, Raj Ashar, Richard Wojcik, Sheri Lewis

Johns Hopkins Applied Physics Laboratory, Laurel, MD, USA.

\section{*Charles Hodanics}

E-mail: charles.hodanics@jhuapl.edu 